\title{
ChemComm
}

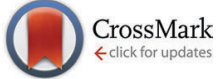

Cite this: Chem. Commun., 2016, 52, 9941

Received 9th May 2016 Accepted 14th July 2016

DOI: $10.1039 / c 6 c c 03895 f$

www.rsc.org/chemcomm

\section{Probing the chemical structure of monolayer covalent-organic frameworks grown via Schiff-base condensation reactions $\dagger$}

\author{
Ya Hu, Niall Goodeal, Ying Chen, Alex M. Ganose, Robert G. Palgrave, \\ Hugo Bronstein and Matthew O. Blunt*
}

\begin{abstract}
Two-dimensional covalent-organic frameworks (2D-COFs) on surfaces offer a facile route to new 2D materials. Schiff-base condensation reactions have proven to be an effective fabrication route for such materials. We present scanning tunneling microscopy (STM) and X-ray photoelectron spectroscopy (XPS) studies of porphyrin 2D-COFs grown at a solid-vapour interface. XPS shows that covalent links between porphyrins consist of a mixture of imines and hemiaminals, a non-conjugated intermediate in the Schiff-base condensation reaction. These results demonstrate that environmental conditions during growth can have an important impact on the chemical composition of Schiff-base 2D-COFs.
\end{abstract}

Two-dimensional (2D) organic materials have far reaching potential for applications in sensing, ${ }^{1 a, b}$ catalysis $^{1 c}$ and molecular electronics. ${ }^{1 d}$ The reversibility of noncovalent interactions mean that they can be used to form 2D molecular structures with long range order. ${ }^{2}$ However, the relative weakness of these interactions often leads to a lack of thermal and chemical stability. Increasing the stability of $2 \mathrm{D}$ materials has been a vital step towards their application.

Two-dimensional covalent organic frameworks (2D-COFs) increase stability by linking molecules using covalent bonds. The use of suitable reaction schemes also opens the possibility of forming fully conjugated $2 \mathrm{D}$ materials. The electronic properties of 2D-COFs are determined by the chemical nature of the links between molecules and the overall structural order. To produce ordered 2D-COFs reactions are required to be reversible during growth. The most commonly used reaction schemes are poly-condensation reactions involving either boronic acids ${ }^{3}$ or amine and aldehyde groups. ${ }^{4-8}$ In recent years both approaches have been used to form 2D-COFs as bulk layered materials ${ }^{4}$ and as individual 2D sheets on underlying substrates. ${ }^{5-8}$ Such surfacesupported 2D-COFs have been grown in various environments

Department of Chemistry, University College London (UCL), 20 Gordon Street, London, WC1H OAJ, UK. E-mail: m.blunt@ucl.ac.uk

$\dagger$ Electronic supplementary information (ESI) available: Detailed experimental and analysis method; additional STM, AFM and XPS data. See DOI: 10.1039/c6cc03895f including ultra-high vacuum $(\mathrm{UHV})^{3 a}$ and at liquid-solid interfaces. ${ }^{5}$

Schiff-base condensation reactions are an effective scheme for the growth of surface-supported 2DCOFs. ${ }^{5-7}$ Studies on the growth of surface-supported imine 2D-COFs at ambient pressures have focused on two approaches: $\mathrm{pH}$ triggered reactions at solid-liquid interfaces; ${ }^{5}$ and temperature induced reactions at solid-vapour interfaces. ${ }^{6,7}$ Imine groups display enhanced stability in relation to boroxine stabilised $2 \mathrm{DCOFs}^{4 a}$ and have the advantage that they form a conjugated link, opening the possibility of producing new, fully conjugated 2D materials.

For 2D-COF growth at solid-vapour interfaces, varying the partial pressure of water controls the reversibility of the condensation reaction. In addition, if one of the reactants is present in the vapour phase then facile reconfiguration of the 2D-COF during growth can occur, thereby preventing kinetic trapping of defects. Wan et al. combined these approaches to produce ordered domains of boroxine ${ }^{3 c}$ and imine stabilised 2D-COFs on highly oriented pyrolytic graphite (HOPG). ${ }^{6 a}$ Since this ground breaking work a variety of imine-stabilised 2D-COFs have been fabricated using solid-vapour reactions. ${ }^{6,7}$ Here we investigate the reaction between either 5,10,15,20-meso-tetra(4-aminophenyl)porphyrin (TAPP) or 5,10,15,20-meso-tetra(4-aminophenyl)porphyrin $\mathrm{Zn}$ (II) (Zn-TAPP) and bezene-1,4-dicarboxaldehyde (BDA) to form surface-supported 2D-COFs on HOPG, see Fig. 1a.

Careful control over the concentration of reactants, temperature and the presence of water can produce ordered monolayer 2D-COFs with domain sizes greater than $100 \mathrm{~nm}$. In addition to structural analysis with STM and atomic force microscopy (AFM), the chemical nature of the 2D-COFs was also investigated using XPS. XPS demonstrates that the covalent links formed by the Schiff-base reaction consist of a mixture of imine and hemiaminal groups, Fig. 1b and c. These results highlight the importance of using complementary spectroscopic techniques in conjunction with STM to analyse 2D-COFs. This approach provides new insights into both the chemistry of 2D-COF formation on surfaces and the role of environmental conditions during growth. 
a)
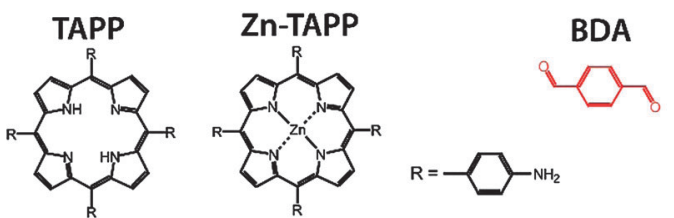

b)

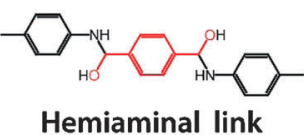

c)

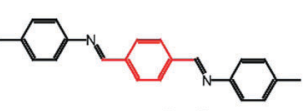

Imine link

Fig. 1 (a) Precursor molecules used in the formation of 2D-COFs $(5,10,15,20$ tetrakis-(4-aminophenyl) porphyrin, TAPP; 5,10,15,20-tetrakis-(4-aminophenyl) porphyrin-zinc(II), Zn-TAPP; and bezene-1,4-dicarboxaldehyde, BDA) (b) Hemiaminal link between porphyrin and BDA. (c) Imine link between porphyrin and BDA.

Synthesis of surface-supported 2D-COFs was performed by drop deposition of $10 \mu \mathrm{l}$ of a toluene solution of TAPP or Zn-TAPP onto freshly cleaved HOPG. Following solvent evaporation samples were placed in sealed glass reaction vessels along with small quantities of BDA and copper(II) sulfate pentahydrate $\left(\mathrm{CuSO}_{4} \cdot 5 \mathrm{H}_{2} \mathrm{O}\right)$. The reaction vessel was then placed in a preheated oven at $130{ }^{\circ} \mathrm{C}$ for $180 \mathrm{~min}$. On removal from the oven the vessels were immediately opened and the samples removed. A detailed experimental procedure is presented in the ESI. $\dagger$

STM characterisation of TAPP and Zn-TAPP 2D-COFs shows an identical 2D square lattice for both systems (Fig. 2a and b). Bright features at the vertices of the 2D-COF structure correspond to the conjugated porphyrin core. Unit cell dimensions for both
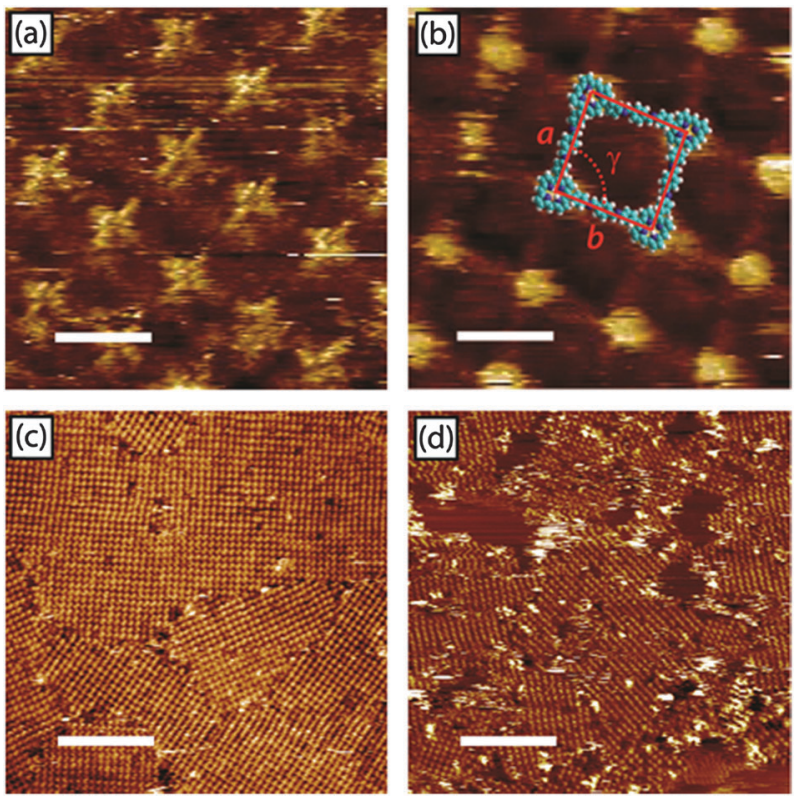

Fig. 2 STM images of porphyrin 2D-COFs on HOPG. (a) and (b) High resolution, drift corrected STM images of TAPP and Zn-TAPP 2D-COFs respectively. (c) and (d) Large area STM images of TAPP and Zn-TAPP 2D-COFs respectively. Imaging parameters: (a) $V_{\mathrm{s}}=0.30 \mathrm{~V}, I_{\mathrm{t}}=5 \mathrm{pA}$; (b) $V_{\mathrm{s}}=-0.50 \mathrm{~V}, I_{\mathrm{t}}=15 \mathrm{pA}$; (c) $V_{\mathrm{s}}=-0.55 \mathrm{~V}, I_{\mathrm{t}}=20 \mathrm{pA}$; (d) $V_{\mathrm{s}}=-0.55 \mathrm{~V}$, $I_{\mathrm{t}}=12 \mathrm{pA}$. Scale bars: (a) $2.5 \mathrm{~nm}$; (b) $2.5 \mathrm{~nm}$; (c) $37.5 \mathrm{~nm}$; (d) $37.5 \mathrm{~nm}$.

2D-COF structures were obtained from drift corrected STM images: $a=b=2.6 \pm 0.2 \mathrm{~nm}$ and $\gamma=89 \pm 3^{\circ}$. These values are in agreement with similar porphyrin 2D-COFs formed by co-deposition of components on HOPG followed by heating under low vacuum, ${ }^{7 b}$ or via a $\mathrm{pH}$ induced reaction at the solid-liquid interface. ${ }^{5 a}$

Large scale STM images (Fig. 2c and d) demonstrate the structural order of the 2D-COFs, with ordered domains for the TAPP 2D-COF in excess of $100 \mathrm{~nm}$. These domains are significantly larger than those observed for porphyrin 2D-COFs grown at solid-liquid interfaces or those grown without one of the components present in the vapour phase. ${ }^{5 a, 7 b}$ These results also represent the first example of a surface-supported 2D-COF grown using a metal porphyrin. Domain sizes observed for the Zn-TAPP 2D-COFs were notably smaller than those seen for the TAPP 2D-COFs. While it is difficult to say with certainty, we suggest that this may be due to differences in solubility between the $\mathrm{Zn}$-TAPP and TAPP. Zn-TAPP had a notably lower solubility in toluene than TAPP, possibly resulting in enhanced aggregation of the $\mathrm{Zn}$-TAPP during solvent evaporation.

The homogeneity of the drop deposited porphyrin films plays a key role in determining the quality of the resulting 2D-COF. AFM was used to investigate the morphology of both the drop deposited porphyrin films and resulting 2D-COFs. The AFM image presented in Fig. 3a shows the HOPG surface after drop deposition of $10 \mu \mathrm{l}$ of TAPP from a $0.008 \mathrm{mg} \mathrm{ml}^{-1}$ toluene solution. This deposition produces a relatively homogeneous distribution of material with a cellular network-like morphology. Cellular networks are commonly observed for the deposition of material from solution following evaporation of a volatile solvent. ${ }^{9}$ After annealing the morphology of the surface changes to that observed in Fig. 2b. The HOPG surface is covered by flat regions of $2 \mathrm{D}-\mathrm{COF}$ with heights in the range $0.5-0.7 \mathrm{~nm}$. Increasing the amount of deposited porphyrin leads to the formation of inhomogeneous films with large $(5-10 \mathrm{~nm})$ 3D aggregates on the surface (ESI, $\dagger$ Fig. S4). This inhomogeneity in the deposited porphyrin hinders the formation of ordered 2D-COFs. The amount of $\mathrm{CuSO}_{4} \cdot 5 \mathrm{H}_{2} \mathrm{O}$ added to the reaction vessel prior to 2D-COF growth also has a significant impact on the 2D-COF morphology (ESI, $\dagger$ Fig. S3).
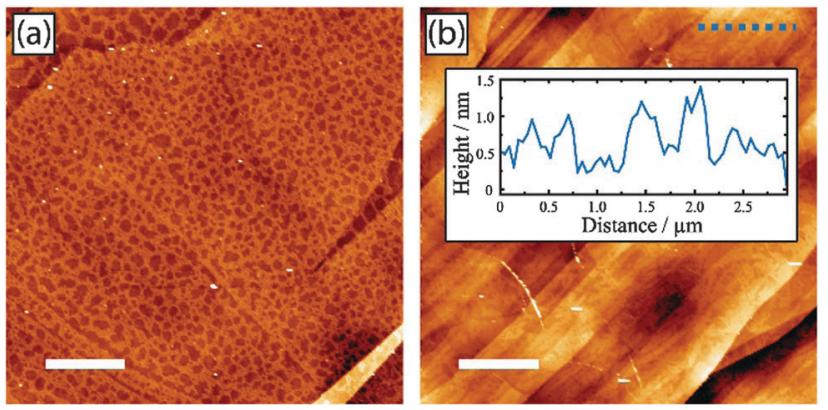

Fig. 3 Tapping mode AFM images of the TAPP/HOPG surface before and after 2D-COF growth. (a) Unreacted TAPP after deposition from toluene: $10 \mu \mathrm{l}$ at $0.008 \mathrm{mg} \mathrm{ml}^{-1}$. (b) HOPG surface after TAPP 2D-COF growth from a sample with the same amount of deposited TAPP. 2D-COF growth parameters: $0.02 \mathrm{mg}$ of $\mathrm{BDA} ; 0.18 \mathrm{~g}$ of $\mathrm{CuSO}_{4} \cdot 5 \mathrm{H}_{2} \mathrm{O} ; 3$ hours at $130{ }^{\circ} \mathrm{C}$. Inset shows a line profile through TAPP 2D-COF domains with heights from $0.5 \mathrm{~nm}$ to $0.7 \mathrm{~nm}$. All images: $10 \mu \mathrm{m}$ with $2 \mu \mathrm{m}$ scale bar. 
XPS was used to investigate the nature of the bonds formed between porphyrin and BDA. For comparison, XPS analysis was performed on unreacted control samples, where TAPP or Zn-TAPP were deposited on HOPG with no heating process applied, in addition to samples with 2D-COF structures. Samples were transferred to UHV and XPS spectra collected for the C1s, O1s and N1s $\mathrm{BE}$ regions. All XPS spectra underwent background subtraction and have been fitted using mixed Gaussian-Lorentzian peak shapes. Full details of the XPS experiments and analysis can be found in the ESI. $\dagger$ As expected, the HOPG surface produces a dominant signal in the $\mathrm{C} 1 \mathrm{~s} \mathrm{BE}$ region. In addition, even clean HOPG samples displayed significant peaks in the O1s region: most likely resulting from contamination (see ESI $\dagger$ ). This made it impossible to use either of these peaks to study the chemical state of the 2D-COFs.

In contrast, the N1s region can be used to study the formation of 2D-COF structures as there is no detectable N1s peak from clean HOPG. Fig. 4a shows the N1s XPS spectra for a drop deposited film of unreacted TAPP on HOPG. The spectrum was modelled with three components in the BE range 397.8-400.0 eV. This is as expected given that the unreacted TAPP molecules contain three distinct nitrogen species. The core of the TAPP molecule contains two electronically inequivalent $\mathrm{N}$ species: one protonated nitrogen $N_{\mathrm{A}}$, corresponding to the light purple component in Fig. $4 \mathrm{a}$ at $400.0 \mathrm{eV}$; and one unprotonated nitrogen $N_{\mathrm{B}}$, corresponding to the light blue component in Fig. 4a at $397.8 \mathrm{eV}$. The difference in $\mathrm{BE}\left(N_{\mathrm{A}}-N_{\mathrm{B}}=2.2 \mathrm{eV}\right)$ and the area ratio $\left(N_{\mathrm{A}} / N_{\mathrm{B}}=1.22\right)$ for these components matches previous XPS studies of porphyrins. ${ }^{10}$ The third major component in Fig. 4a, coloured dark purple at $399.4 \mathrm{eV}$, corresponds

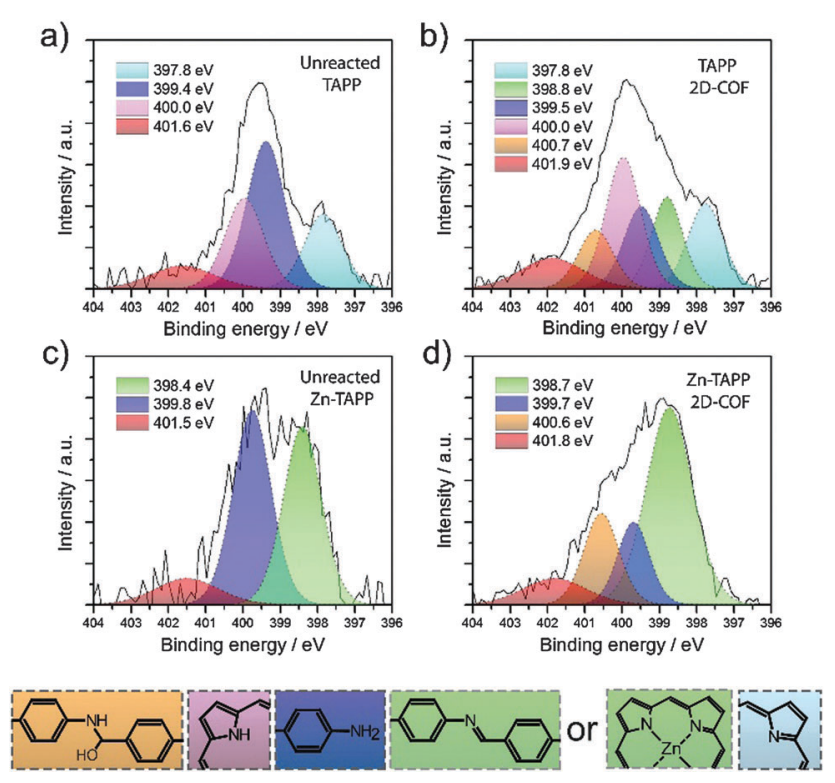

Fig. 4 N1s XPS spectra for: (a) unreacted TAPP; (b) TAPP 2D-COF; (c) unreacted Zn-TAPP; and (d) Zn-TAPP 2D-COF. The black lines represent experimental data after background subtraction. The coloured components correspond to Gaussian-Lorentzian functions used to fit the data. The graph legends show the BE associated with each component. The schematic at the bottom of the figure shows the molecular configurations of the different $\mathrm{N}$ species. to unreacted amine groups. An additional much less intense component is observed at $401.6 \mathrm{eV}$, corresponding to the $\mathrm{BE}$ range (401-402 eV) where satellite peaks are commonly observed for porphyrins. ${ }^{10}$

Following 2D-COF growth the N1s XPS spectra changes significantly, and is modelled with several new components, see Fig. 4b. Components associated with the nitrogen species within the porphyrin core (397.8 eV and $400.0 \mathrm{eV}$ ) remain largely unchanged. This is as expected given that these species are not directly involved in the Schiff-base condensation reaction. In contrast, the intensity of the component associated with amine groups $(399.5 \mathrm{eV})$ reduces significantly. Meanwhile, two new components are introduced at $400.7 \mathrm{eV}$ (orange) and $398.8 \mathrm{eV}$ (green). The component at $398.8 \mathrm{eV}$ corresponds to the BE expected for an imine nitrogen formed by a Schiff-base condensation reaction, Fig. 1c. ${ }^{11}$ While the component at $400.7 \mathrm{eV}$ corresponds to the $\mathrm{BE}$ expected for a nitrogen in a hemiaminal structure, as shown in Fig. 1b. ${ }^{11}$ Hemiaminals are known intermediate states in the Schiff-base condensation reaction and have previously been identified in solution by Raman spectroscopy, mass spectroscopy and nuclear magnetic resonance (NMR). ${ }^{12 a-c}$

Fig. 4c and d show N1s XPS spectra for Zn-TAPP. The unreacted control sample is modelled with two components: one at $398.4 \mathrm{eV}$, consistent with the four $\mathrm{N}$ atoms coordinated to the central $\mathrm{Zn}$; and one at $399.8 \mathrm{eV}$, consistent with unreacted amine groups. As expected the area of these two components is approximately equal. Following 2D-COF growth an additional component appears at $400.6 \mathrm{eV}$ consistent with hemiaminal formation. The component associated with unreacted amine decreases in intensity, while the one associated with imines increases. It should be noted that the $\mathrm{BE}$ of imine and $\mathrm{Zn}$ coordinated $\mathrm{N}$ are almost identical making it impossible to separate the contributions of these species. ${ }^{10,11}$

Hemiaminal groups have been observed by XPS in solution grown 1D Schiff-base polymers. ${ }^{11}$ However, the results presented here are the first evidence that hemiaminal covalent links are present in monolayer 2D-COFs grown via Schiff-base condensation at a solid-vapour interface. The XPS results provide a strong indication that the porphyrin 2D-COFs contain a mixture of both imine and hemiaminal links between the porphyrin and BDA components. The fact that the N1s XPS component associated with amine does not completely disappear for the 2D-COF samples suggests that significant numbers of unreacted amines also remain on the surface after 2D-COF growth. Amine groups could be present at domain edges, defects in the 2D-COF or within 3D clusters of unreacted TAPP molecules as observed via AFM (ESI, $\dagger$ Fig. S4).

Schiff-base condensation at a solid-vapour interface is one of the most effective approaches for the growth of ordered 2D-COF monolayers. Consistent with previous studies ${ }^{11}$ we do not observe any features in STM images that differentiate between hemiaminal and imine links. This structural similarity demonstrates the importance of employing spectroscopic analysis, such as XPS, to probe the chemical nature of linking covalent bonds. In order to produce $2 \mathrm{D}$ structures with desirable properties, such as conjugated 2D-COFs, the nature of chemical links between molecules needs to be accurately controlled. These results pose important questions about how the environment used during the growth of 
Schiff-base 2D-COFs influences the chemical structure of these materials.

The conversion of hemiaminal to imine is itself a reversible reaction. Standard routes for imine synthesis employ drying techniques to help increase imine yield. ${ }^{13}$ To investigate the influence that water has on 2D-COF growth XPS data was collected for TAPP 2D-COF samples grown with varying amounts of $\mathrm{CuSO}_{4} \cdot 5 \mathrm{H}_{2} \mathrm{O}$ present (ESI, $\dagger$ Fig. S9-S13 and Table S3). When no $\mathrm{CuSO}_{4} \cdot 5 \mathrm{H}_{2} \mathrm{O}$ was present the $\%$ contribution of hemiaminal to the overall N1s XPS signal decreased (down to $3.1 \%$ from $13.4 \%$ ) while that of imine increased (up to $35.8 \%$ from $18.6 \%$ ). However, it is important to note that STM analysis of the sample grown with no added $\mathrm{CuSO}_{4} \cdot 5 \mathrm{H}_{2} \mathrm{O}$ showed no ordered $2 \mathrm{D}-\mathrm{COF}$ structure. This absence of ordered structure suggests that under these conditions the Schiff-base reaction lacks sufficient reversibility for stable 2D-COF growth.

For the ordered 2D-COFs studied in Fig. 2-4 the reaction equilibrium has most probably been held at a position that favours the formation of a significant quantity of hemiaminal linkages. This is consistent with the sustained presence of water during 2D-COF growth. The presence of water maintains the equilibrium of the reaction biased towards hemiaminal formation. The fact that the hemiaminal links remain after the growth process is stopped may be related to the experimental approach used. The rapid removal of the sample from the reaction vessel, before it has cooled, effectively quenches the sample and locks-in the chemical composition of the growing 2D-COF. This rapid quenching produces the mixture of hemiaminal and imine links observed in the final structure.

As shown in the ESI $\dagger$ (Tables S1 and S2) the \% contributions of imine and hemiaminal to the N1s XPS signal vary between the TAPP and Zn-TAPP 2D-COF samples. Given the limited data it is impossible to attribute this variation to either a direct effect of the presence of Zn, or simply to statistical fluctuation. Further studies will investigate the potential effect that $\mathrm{Zn}$ and other central metal atoms have on 2D-COF formation along with the influence of environmental factors during growth.

In summary, we have fabricated highly ordered porphyrin 2D-COFs supported on HOPG surfaces. These 2D-COFs are stabilised via a Schiff-base condensation reaction between BDA and porphyrins. The morphology and chemical compositions of these structures were investigated using a combination of scanning probe microscopy and XPS. The 2D-COFs contained ordered domains in excess of $100 \mathrm{~nm}$ in size. In addition, we demonstrated the first example of a surface-supported 2D-COF formed using a metal containing porphyrin linked via a Schiff-base condensation reaction: namely Zn-TAPP. XPS analysis of the 2D-COFs highlighted that the covalent links formed between components consist of a mixture of imine and hemiaminal groups. These results show the importance of utilizing complementary techniques to probe both the overall morphology and the chemical structure of 2D-COFs. Previous studies have shown that the growth environment, particularly the presence of water, has important implications for the structural order of 2D-COFs.
The results presented here show that the presence of water can also impact on the nature of the bonds formed between components. The exact nature of the links formed between molecular components plays a significant role in determining the electronic band structure of 2D-COF materials. For example, imine links between BDA and TAPP molecules produce a fully conjugated 2D-COF structure $^{7 b}$ whereas the presence of hemiaminal linkages breaks this conjugation. The potential of porphyrin 2D-COFs to act as components in sensors and nano-electronic devices will only be fully realized when control over both the morphology and the chemical composition of these novel materials is obtained.

This study was supported by an Engineering and Physical Sciences Research Council First Grant (EP/N021789/1) and a Marie Curie Career Integration Grant (618777).

\section{Notes and references}

1 (a) J. Liu, Z. Liu, C. J. Barrow and W. Yang, Anal. Chim. Acta, 2015, 859, 1-19; (b) D. Monti, S. Nardis, M. Stefanelli, R. Paolesse, C. Di Natale and A. D'Amico, J. Sens., 2009, 2009, 1-10; (c) S. Nakagaki, G. K. Ferreira, G. M. Ucoski and K. A. Dias de Freitas Castro, Molecules, 2013, 18, 7279-7308; (d) J. Yang, D. Yan and T. S. Jones, Chem. Rev., 2015, 115, 5570-5603.

2 A. Ciesielski, C. A. Palma, M. Bonini and P. Samori, Adv. Mater., 2010, 22, 3506-3520.

3 (a) N. A. A. Zwaneveld, R. Pawlak, M. Abel, D. Catalin, D. Gigmes, D. Bertin and L. Porte, J. Am. Chem. Soc., 2008, 130, 6678-6679; (b) J. F. Dienstmaier, A. M. Gigler, A. J. Goetz, P. Knochel, T. Bein, A. Lyapin, S. Reichlmaier, W. M. Heckl and M. Lackinger, ACS Nano, 2011, 5, 9737-9745; (c) C. Z. Guan, D. Wang and L. J. Wan, Chem. Commun., 2012, 48, 2943-2945.

4 (a) S. Kandambeth, D. B. Shinde, M. K. Panda, B. Lukose, T. Heine and R. Banerjee, Angew. Chem., Int. Ed., 2013, 52, 13052-13056; (b) H. Wei, S. Chai, N. Hu, Z. Yang and L. Wang, Chem. Commun., 2015, 51, 12178-12181; (c) Y. Wu, H. Xu, X. Chen, J. Gao and D. Jiang, Chem. Commun., 2015, 51, 10096-10098.

5 (a) R. Tanoue, R. Higuchi, N. Enoki, Y. Miyasato, S. Uemura, N. Kimizuka, A. Z. Stieg, J. K. Gimzewski and M. Kunitake, ACS Nano, 2011, 5, 3923-3929; (b) L. R. Xu, X. Zhou, Y. X. Yu, W. Q. Tian, J. Ma and S. B. Lei, ACS Nano, 2013, 7, 8066-8073; (c) L. R. Xu, X. Zhou, W. Q. Tian, T. Gao, Y. F. Zhang, S. B. Lei and Z. F. Liu, Angew. Chem., Int. Ed., 2014, 53, 9564-9568.

6 (a) X. H. Liu, C. Z. Guan, S. Y. Ding, W. Wang, H. J. Yan, D. Wang and L. J. Wan, J. Am. Chem. Soc., 2013, 135, 10470-10474; (b) X. H. Liu, C. Z. Guan, Q. N. Zheng, D. Wang and L. J. Wan, J. Chem. Phys., 2015, 142, 101905.

7 (a) Y. X. Yu, J. Sun and S. B. Lei, J. Phys. Chem. C, 2015, 119, 16777-16784; (b) X. L. Sun, L. X. Fan, X. Zhou, W. Q. Tian, Z. X. Guo, Z. B. Li, X. K. Li and S. B. Lei, Chem. Commun., 2015, 51, 5864-5867.

8 X. H. Liu, C. Z. Guan, D. Wang and L. J. Wan, Adv. Mater., 2014, 26, 6912-6920.

9 H. Alhummiany, S. Jarvis, R. A. J. Woolley, A. Stannard, M. Blunt and P. Moriarty, J. Mater. Chem., 2011, 21, 16983-16989.

10 V. N. Nemykin, P. Galloni, B. Floris, C. D. Barrett, R. G. Hadt, R. I. Subbotin, A. G. Marrani, R. Zanoni and N. M. Loim, Dalton Trans., 2008, 4233-4246.

11 M. Di Giovannantonio, T. Kosmala, B. Bonanni, G. Serrano, N. Zema, S. Turchini, D. Catone, K. Wandelt, D. Pasini, G. Contini and C. Goletti, J. Phys. Chem. C, 2015, 119, 19228-19235.

12 (a) A. Cooper, S. F. Dixon, M. A. Nutley and J. L. Robb, J. Am. Chem. Soc., 1987, 109, 7254-7263; (b) T. Q. Zhang, W. Zhou, W. Jin, Q. H. Jin and H. W. Chen, Microchem. J., 2013, 108, 18-23; (c) A. L. Upthagrove and W. L. Nelson, Drug Metab. Dispos., 2001, 29, 1114-1122.

13 C. D. Meyer, C. S. Joiner and J. F. Stoddart, Chem. Soc. Rev., 2007, 36, 1705-1723. 